Bull. Chem. Soc. Ethiop. 2020, 34(2), 277-284.

ISSN 1011-3924

(c) 2020 Chemical Society of Ethiopia and The Authors

Printed in Ethiopia

DOI: https://dx.doi.org/10.4314/bcse.v34i2.6

\title{
CONSTRUCTION OF POROUS CARBON FOR THE HIGHLY EFFICIENT VISIBLE LIGHT-DRIVEN DEGRADATION METHYL VIOLET
}

\author{
Chuncheng $\mathrm{Shi}^{1}$, Xiaoxiong Wang ${ }^{2}$, Ying Dong ${ }^{3}$, Wuxin $\mathrm{Hu}^{4 *}$, Ying $\mathrm{Li}^{3}$, Ying $\mathrm{Pan}^{3 *}$, Yuzhi Qiu ${ }^{3}$ \\ and Jianqiang $\mathrm{Liu}^{3}$ \\ ${ }^{1}$ Department of Pharmacy, School of Medicine, Xi'an International University, Xi'an, 710077, \\ Shaanxi, China \\ ${ }^{2}$ School of Civil and Environmental Engineering, Shenzhen Polytechnic, Shenzhen, 518055, \\ China \\ ${ }^{3}$ Key Laboratory of Research and Development of New Medical Materials of Guangdong \\ Medical University, School of Pharmacy, Guangdong Medical University, Dongguan, 523808, \\ China \\ ${ }^{4}$ School of Public Health and Management, Wenzhou Medical University, Wenzhou 325035, \\ China
}

(Received April 29, 2020; Revised October 10, 2020; Accepted October 19, 2020)

ABSTRACT. A series of nanoporous carbons (NPC) Fe-C/N-900 and C/N-900 have been synthesized from onestep carbonization of 5,10,15,20-tetrakis(4-carboxyphenyl)porphyrin-Fe (Fe-TCPP) and TCPP, respectively and employed as photocatalyst for the degradation of organic dye methyl violet (MV) under UV irradiation. The optimized Fe-C/N-900 (carbonized at $900{ }^{\circ} \mathrm{C}$ for $2 \mathrm{~h}$ ) exhibited an optimal performance in MV degradation. The photodegradation capacity of $\mathrm{Fe}-\mathrm{C} / \mathrm{N}-900$ has been observed to be higher than that of $\mathrm{C} / \mathrm{N}-900$. The photodegradation ability of Fe-C/N-900 as a function of initial MV concentration, catalysis dosage, and $\mathrm{pH}$ has been also investigated. The $\mathrm{Fe}-\mathrm{C} / \mathrm{N}-900$ material showed no apparent loss in $\mathrm{MV}$ degradation after four cycles. These features reveal that $\mathrm{Fe}-\mathrm{C} / \mathrm{N}-900$ may be a promising degradant for dyes removal from water.

KEY WORDS: Photocatalysis, Nanoporous carbons, Methyl violet, carbonization

\section{INTRODUCTION}

The wastewater bodies comprising of organic dyes are nowadays the most frequently detected environmental pollutants due to exponential rise in the consumption of these dyes because of the continuous growth in population [1-3]. The rise in such pollutant is the reason of immense concern because they lead to adverse effects on aquatic life and humans [4-6]. In view of this several methods and techniques, such as adsorption, biodegradation, and chemical oxidation have been used to treat the dyes in the wastewater discharge. Since these methods are having some limitations, therefore, new highly efficient methods with wide applicability and simple in design are an urgent need of the hour to cope-up in a better way with this increasingly serious pollution issue. Fujishima et al. in their pioneer work on the process of changing light energy to chemical energy by photocatalyst had used $\mathrm{TiO}_{2}$ and $\mathrm{CdS}$ as the representative of photocatalysts for the degradation of the aromatic dyes [7-8].

Recently, advanced porous materials have created attention of the materials chemists because they offer myriad of applications [9]. Using high temperature pyrolysis, ZIF-8 had been converted to highly porous carbons with acidic and basic functionalities with high specific surface area [10]. This was employed in liquid phase adsorption for the removal of drugs and organic dye (methylene blue) from water [11]. However, utilization of this material was not investigated in detail. Therefore, more detailed studies of the application of nitrogen-containing carbons obtained from MOFs and hybrid materials are required. This is because, such materials are innately fascinating because of their high porosity and hydrophobic nature.

*Corresponding author. E-mail: ying-p@163.com; hwx@wmu.edu.cn

This work is licensed under the Creative Commons Attribution 4.0 International License 
In the investigation presented herein, nanoporous carbons (NPC) have been derived through one-step carbonization of 5,10,15,20-tetrakis(4-carboxyphenyl)porphyrin-Fe (Fe-TCPP) and 5,10,15,20-tetrakis (4-carboxyphenyl)porphyrin (TCPP). These newly synthesized materials have been assessed towards the visible-light degradation of aromatic dyes. The results of their comparative photocatalytic performances are presented.

\section{EXPERIMENTAL}

\section{General considerations}

All reagents obtained from commercial sources and used without further purification. Scanning electron microscopy (SEM) has been carried out on FEI Quanta 400 FEG field emission scanning electron microscope. Nitrogen adsorption-desorption measurements were performed at $77 \mathrm{~K}$ in a liquid nitrogen bath on a Micromeritics ASAP 3020 analyzer. Samples were degassed at $150{ }^{\circ} \mathrm{C}$ for $10 \mathrm{~h}$ in a gas vacuum system prior to analysis. The Brunauer-Emmett-Teller (BET) method was used to calculate the specific surface areas $\left(S_{B E T}\right)$. Pore size distributions were obtained from the $\mathrm{N}_{2}$ adsorption isotherms using density functional theory (DFT) method. The photocatalytic activity studies were carried out using Shimadzu UV-Vis 2501PC spectrophotometer.

Syntheses of $\mathrm{C} / \mathrm{N}-900$ and $\mathrm{Fe}-\mathrm{C} / \mathrm{N}-900$

In a typical carbonization process, $500 \mathrm{mg}$ of 5,10,15,20-tetrakis(4-carboxyphenyl)porphyrin-Fe (Fe-TCPP) or 5,10,15,20-tetrakis(4-carboxyphenyl)porphyrin (TCPP) was transferred into a ceramic boat and placed in a temperature programmed furnace. The furnace was heated to 900 ${ }^{\circ} \mathrm{C}$ with a ramp rate of $5{ }^{\circ} \mathrm{C} \mathrm{min}{ }^{-1}$ and kept for $2 \mathrm{~h}$ under flowing argon atmosphere, and cooled naturally to room temperature. The resulting nanoporous carbon materials were labeled as $\mathrm{Fe}-$ C/N-900and C/N-900, respectively.

\section{Photocatalytic method}

The photocatalytic reactions were performed as follows: The samples C/N-900 or Fe-C/N-900 $(20 \mathrm{mg}$ ) was dispersed in $50 \mathrm{~mL}$ aqueous solution containing methyl violet (MV), methyl blue $(\mathrm{MB})$ and rhodamine $\mathrm{B}(\mathrm{Rh} \mathrm{B})(20 \mathrm{mg} / \mathrm{L})$. The mixture was stirred in dark for $30 \mathrm{~min}$ to ensure the establishment of adsorption-desorption equilibrium. The photocatalytic degradation processes of $\mathrm{MV}, \mathrm{MB}$ and $\mathrm{Rh} \mathrm{B}$ were conducted on an XPA-7 type photochemical reactor equipped with a 100 WLED lamp (mean wavelength $365 \mathrm{~nm}$ ). During experiment, aliquots of $5.0 \mathrm{~mL}$ were taken out at specified time intervals and the clear solution was separated through centrifugation and then subsequently analyzed by using a UV-visible spectrophotometer.

\section{RESULTS AND DISCUSSION}

\section{Characterization of the catalysis}

The carbon materials viz. $\mathrm{C} / \mathrm{N}-900$ and $\mathrm{Fe}-\mathrm{C} / \mathrm{N}-900$ were prepared by controlling the carbonization temperature at $900{ }^{\circ} \mathrm{C}$. By optimizing the carbonization time period, the desired carbon samples $\mathrm{C} / \mathrm{N}-900$ and $\mathrm{Fe}-\mathrm{C} / \mathrm{N}-900$ were obtained with a short hold time of $2 \mathrm{~h}$. SEM images demonstrate that the obtained carbon materials are micron-sized carbon blocks with a compact surface (Figure 1). The morphology of C/N-900 and Fe-C/N-900 change noticeably upon pyrolysis (irrespective of treatment time), which is similar to previous works [12]. The $\mathrm{N}_{2}$ sorption isotherms of the sample obtained from carbonizing Fe-TCPP precursors is of type IV (Figure 2), which is indicative of the coexistence of micropore and mesopore. The results 
obtained from the isotherms are in good agreement with the compact morphologies observed in SEM images. The surface area and pore volume of Fe-C/N-900 is $214.6166 \mathrm{~m}^{2} / \mathrm{g}$ and 0.17502 $\mathrm{cm}^{3} / \mathrm{g}$, respectively, which is higher in comparison to C/N-900 with $14.3274 \mathrm{~m}^{2} / \mathrm{g}$ and $0.00857 \mathrm{~cm}^{3} / \mathrm{g}$.

\section{$U V$-Vis diffuse reflectance spectroscopy (DRS)}

The optical properties of the photocatalysts were investigated by DRS. The optical band gap of the samples were estimated using the Tauc plot in which the plot of $(\alpha h v)^{2}$ versus the energy of the absorbed light gives band gaps of samples. The $E_{\mathrm{g}}$ value is determined by measuring the $x$ axis intercept of an extrapolated tangential line from the linear region of the curve. The $E_{\mathrm{g}}$ values provide insight regarding the light absorption and photocatalytic efficiency of the samples [13]. The band gaps of Fe-TCPP, C/N-900 and Fe-C/N-900 were estimated to be 2.78 $\mathrm{eV}, 3.51 \mathrm{eV}$ and $2.47 \mathrm{eV}$, respectively (Figure 3). The larger the $E_{\mathrm{g}}$ is, the smaller the degradation rate in the same time.

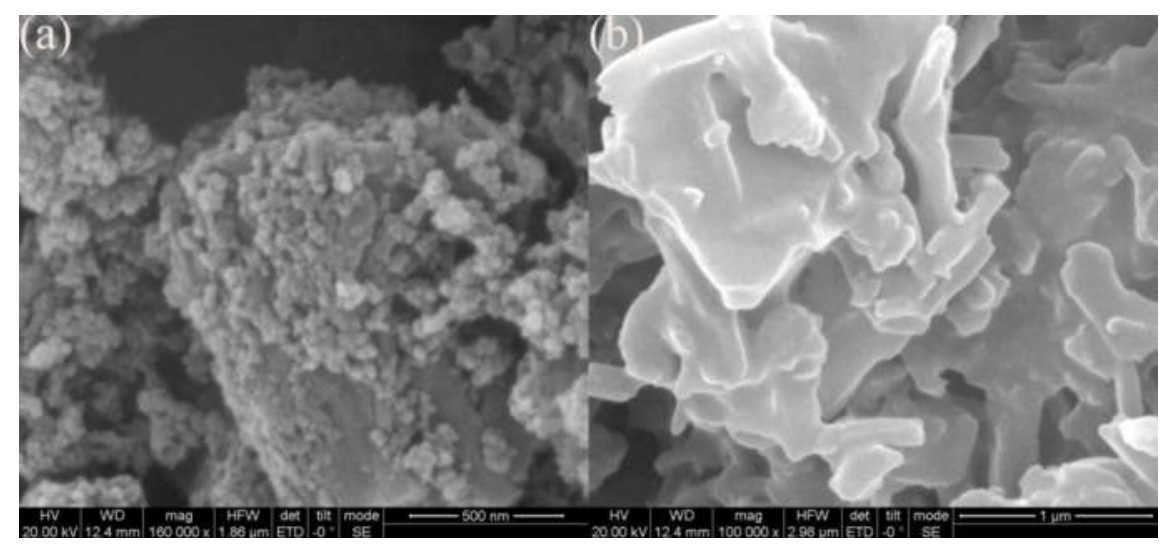

Figure 1. SEM images of (a) Fe-C/N-900 and (b) C/N-900.
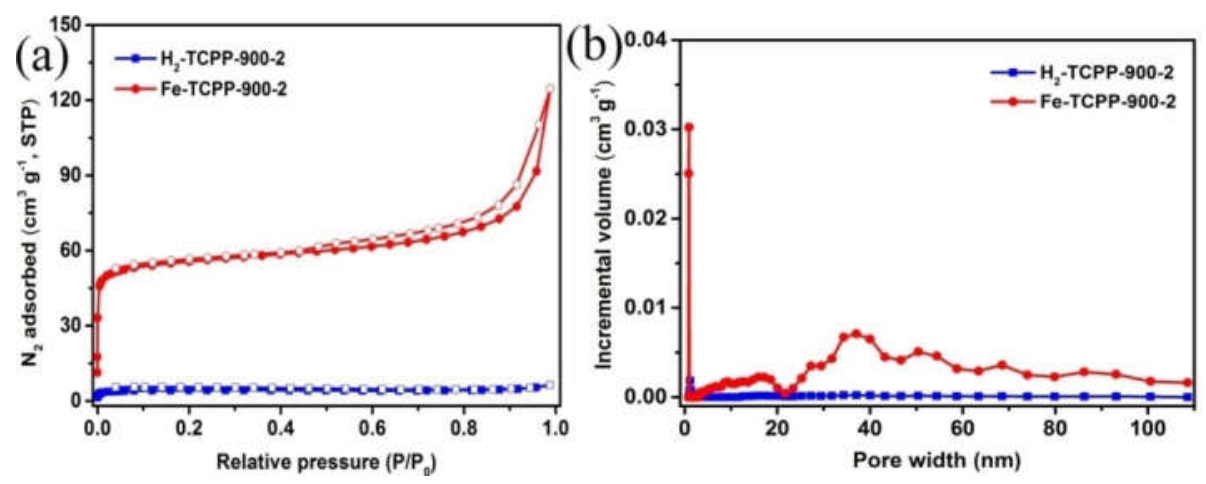

Figure 2. $\mathrm{N}_{2}$ adsorption-desorption isotherms of samples. 


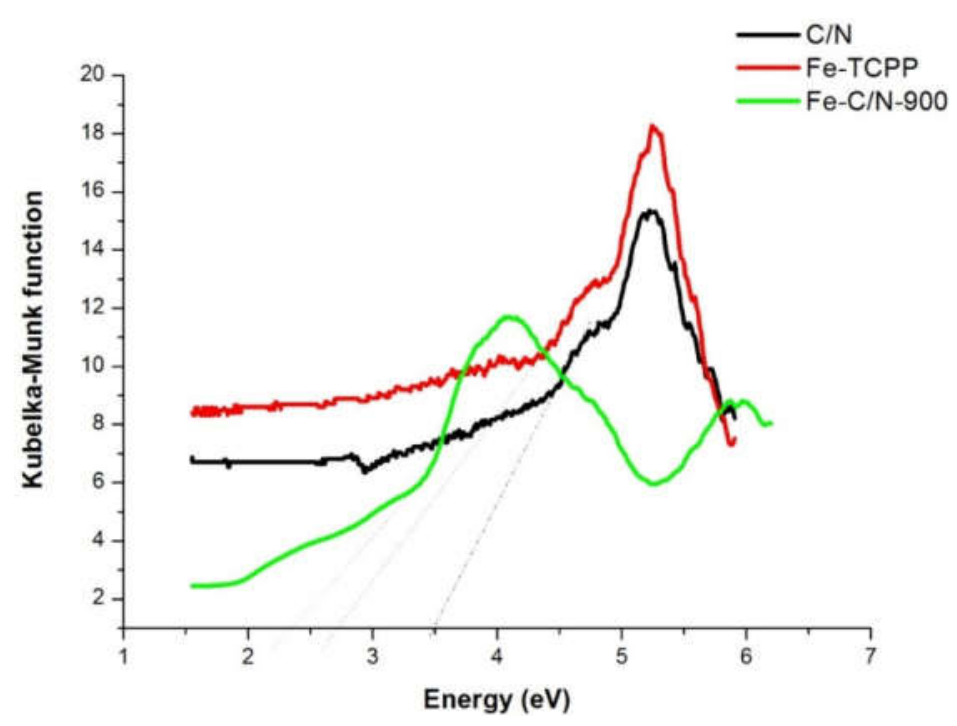

Figure 3. The band gaps of materials.

\section{Photocatalysis}

Photocatalytic degradation of methylene blue (MB), methyl violet (MV) and rhodamine $\mathrm{B}$ (Rh B) by $\mathrm{Fe}-\mathrm{C} / \mathrm{N}-900$ were conducted to investigate the efficiency of photocatalyst. The photocatalytic activities of Fe-C/N-900 were monitored by the variation of the color in there action medium by recording the maximum absorbance intensity. The absorption band of dyes were observed to drop on prolonging the reaction time in presence of $\mathrm{Fe}-\mathrm{C} / \mathrm{N}-900$. In presence of $\mathrm{Fe}-\mathrm{C} / \mathrm{N}-900$, within 25 min under UV irradiation the percentage photodegradation of $\mathrm{Rh} \mathrm{B}$, $\mathrm{MB}$, and MV are 19.55, 46.33 and 56.99\%, respectively (Figure 4). Meanwhile, the percentage photodegradation of MV was $\sim 27.08,56.99$ and $55.61 \%$ when pure $\mathrm{C} / \mathrm{N}-900, \mathrm{Fe}-\mathrm{C} / \mathrm{N}-1000$ and Fe-TCPP were utilized as the photocatalysts. The results indicate that $\mathrm{Fe}-\mathrm{C} / \mathrm{N}-900$ displays better photocatalytic performance compared to pure C/N-900 and Fe-TCPP. The better performance of $\mathrm{Fe}-\mathrm{C} / \mathrm{N}-900$ can be credited to formation of the unique hetero junctions [14]. The $\mathrm{C}$ doped cubic $\mathrm{ZnO}$ was also obtained by using $\mathrm{ZIF}-8$ as the precursor. Under visible-light irradiation, the $\mathrm{C} 350-400$ (5.4\% $\mathrm{C}$ doping) shows a 1.2-fold, 1.7-fold, and 1.8-fold higher photoactivity than $\mathrm{C} 350-450$ (3.6\% C doping), $\mathrm{C}-\mathrm{ZnO}(2.7 \% \mathrm{C}$ doping), and $\mathrm{C} 400(1.9 \% \mathrm{C}$ doping), respectively, in $\mathrm{Rh} \mathrm{B}$ degradation, which results from the enhanced electron-hole separation and optical absorption/mass transfer. However, the photocatalytic efficiency is not very satisfactory and is lower than that of the title materials [15].

Influence of the operating parameters on the reaction

The catalyst dosage is an important parameter for catalytic activity; the Fe-C/N-900 catalyst with varied concentrations of 5, 10,15, 20,30 mg/L was added to MV (Figure 5a and 5d). With increase in the catalyst dosage, the degradation rate was not observed to increase obviously. When catalyst dosage is increased, more electrons will be produced and hence more photogenerated electrons will be captured. However, this phenomenon was witnessed only at low dosages. Hence, when the dosage of $\mathrm{Fe}-\mathrm{C} / \mathrm{N}-900$ was increased to $30 \mathrm{mg} / \mathrm{L}$, the dosage effect on the photocatalytic performance can be ignored. The almost neutral effect of the high dosage of $\mathrm{Fe}-\mathrm{C} / \mathrm{N}-900$ can be attributed to the high concentration of the catalyst which remains suspended in solution and causes part of visible light to be lost by scattering effect [15]. 

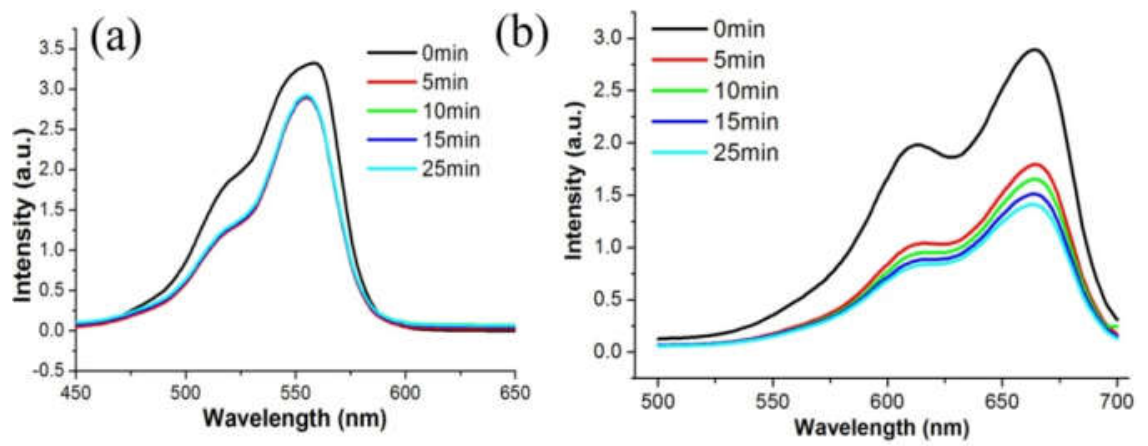

(c)

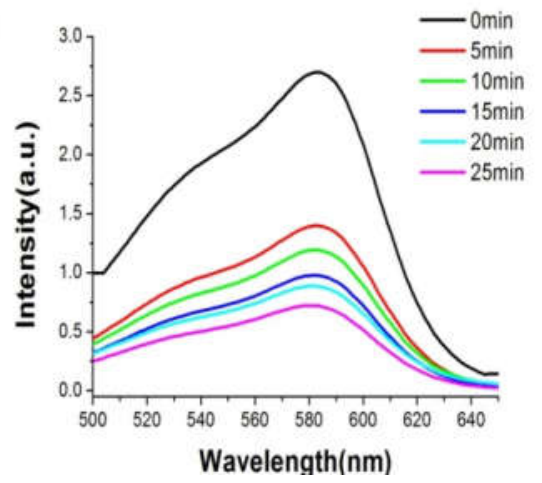

Figure 4. UV-vis absorption spectraof the Rh B, MB and MV solutions degraded by $\mathrm{Fe}-\mathrm{C} / \mathrm{N}$ 900 under UV irradiation at different time intervals for (a), (b) and (c), respectively.

The $\mathrm{pH}$ value also plays an important role during photocatalytic reaction because it affect the surface charges of photocatalytic materials as well as pollutants [15]. Hence, HOAc $(0.25$ $\mathrm{M})$ or $\mathrm{NaOH}(0.25 \mathrm{M})$ solution was employed in adjusting the $\mathrm{pH}$ of MV solution to probe the effect of variation in $\mathrm{pH}$ on photocatalytic property of material. The initial $\mathrm{pH}$ of $\mathrm{MV}$ ranged in 6-8. The optimal degradation rate of $\mathrm{MV}$ was $63.91 \%$ at $\mathrm{pH}$ value of 7.5 while the photodegradation of MV suppressed to some extent at other $\mathrm{pH}$ values (Figure $5 \mathrm{~b}$ and $5 \mathrm{e}$ ). The photocatalytic activity was also inhibited under strong alkaline condition although the photocatalysis during first $5 \mathrm{~min}$ was accelerated. Such acceleration could be ascribed to the negatively charged surface state of $\mathrm{Fe}-\mathrm{C} / \mathrm{N}-900$ which takes benefit for the photo-generated holes migrating to the surface for degrading pollutants [16].

For the purpose of studying the effects of MV concentration on photocatalytic performance, the initial concentration of MV was set as $2 \times 10^{-5}, 4 \times 10^{-5}, 4 \times 10^{-5}$ and $5 \times 10^{-5} \mathrm{mg} / \mathrm{L}$ (Figure $5 \mathrm{c}$ and $5 \mathrm{f}$ ). It was found that when the initial concentration of MV was $2 \times 10^{-5}, 4 \times 10^{-5}, 4 \times 10^{-5}$ and $5 \times 10^{-5} \mathrm{mg} / \mathrm{L}$, the corresponding MV degradation rate was $58.72,70,50.14,41.88 \%$ and $39.80 \%$, respectively. It could be found that the initial concentration of $\mathrm{MV}$ has little impact on the photodegradation process but high concentration of MV may inhibit the photocatalytic efficiency. It could be attributed to the light penetration and the competition between intermediate and dye molecules. It is speculated that higher concentration of dyes could inhibit the light penetration and which may result in less number of photon capturing by the catalyst [17-18]. 


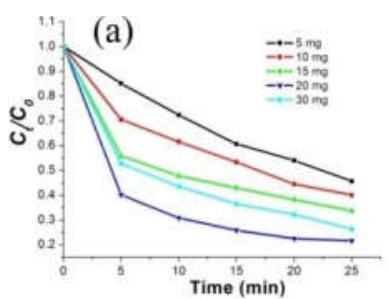

(d)

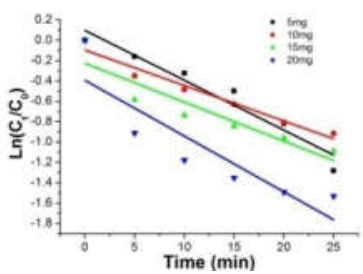

(b)

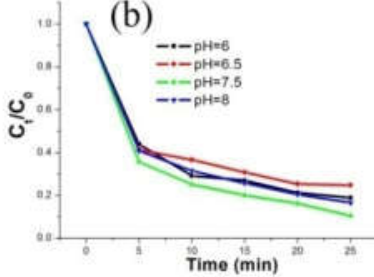

(e)

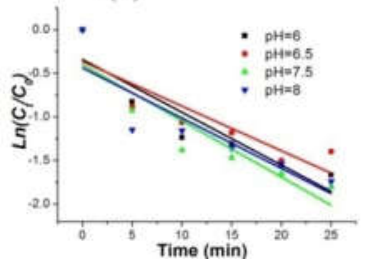

(c)

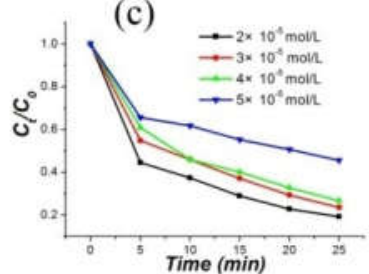

(f)

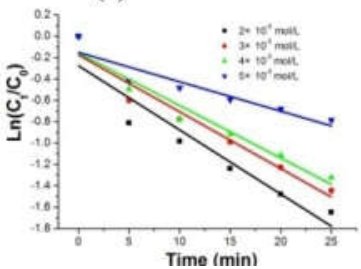

Figure 5. (a)-(c) Curves of absorbance of the MV solution degraded by Fe-C/N-900 under UVvis light irradiation and (d)-(f) linear-log plot as a function of visible light irradiation time in the presence of $\mathrm{Fe}-\mathrm{C} / \mathrm{N}-900$ under different conditions.

(a)

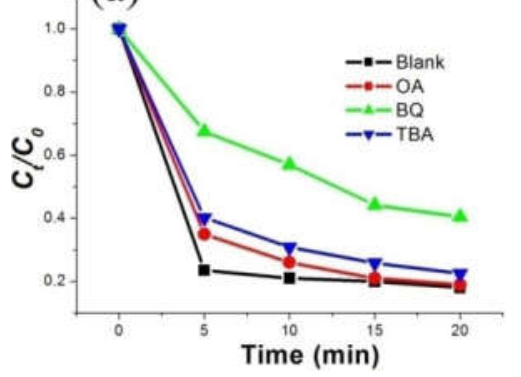

(b)

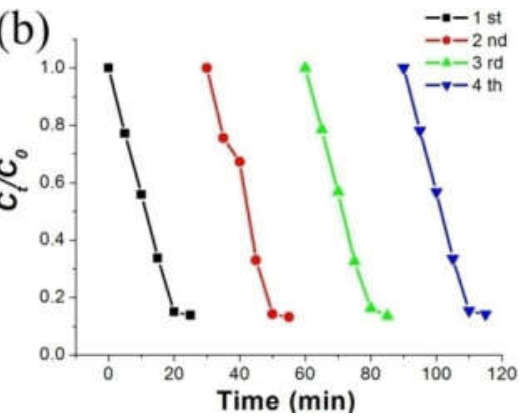

Figure 6. (a) photodegradation of the MV solution over Fe-C/N-900 in the different scavenger solutions; (b) cycling runs of the photocatalytic degradation of MV for Fe-C/N-900.

Photocatalytic mechanism study

To investigate the plausible photocatalytic mechanism, the photodegradation of MV was carried out in the presence of tertiary butyl alcohol (TBA), benzoquinone (BQ) and ammonium oxalate (AO) which act as $\cdot \mathrm{OH}, \mathrm{O}_{2} \cdot{ }^{-}$and holes $\left(\mathrm{h}^{+}\right.$) radicals quenchers, respectively (Figure 6a) [19-20]. The photocatalytic reactions performed in presence of these quenchers indicated that BQ was capable of declining the photodegradation of $\mathrm{MV}$ in the presence of $\mathrm{Fe}-\mathrm{C} / \mathrm{N}-900$. It was observed that the relevant rate constants for the decomposition of $\mathrm{MV}$ in presence of $\mathrm{Fe}-\mathrm{C} / \mathrm{N}-$ 900 decreased in the presence of BQ under UV-Vis light. Therefore, the results of $\mathrm{O}_{2}{ }^{-}$ quenching experiments suggest that the photodegradation of MV by the photocatalyst $\mathrm{Fe}-\mathrm{C} / \mathrm{N}$ 900 is dominated by $\mathrm{O}_{2} \cdot{ }^{-}$radical anion. In addition, the activity of the recycled catalyst is very important for determining the performance of the photocatalyst. The solid samples were collected by centrifugation and drying after the recycled experiments, the recycled $\mathrm{Fe}-\mathrm{C} / \mathrm{N}-900$ exhibited similar photocatalytic performance when compared to the pristine Fe-C/N-900 (Figure 
6b) [21]. Thus, in can be shown that $\mathrm{Fe}-\mathrm{C} / \mathrm{N}-900$ is stable during photocatalysis and it can be used as stable photocatalyst for photodegradation of organic dyes [22-25].

\section{CONCLUSION}

In the presented work, the $\mathrm{Fe}-\mathrm{C} / \mathrm{N}-900$ and $\mathrm{C} / \mathrm{N}-900$ materials had been successfully synthesized using a facile one-step carbonization method and employed as catalyst for MV degradation. The enhancement of the photocatalytic activity could be attributed to more $\mathrm{Fe}$ active sites on the composite surface in $\mathrm{Fe}-\mathrm{C} / \mathrm{N}-900$. Besides, various reaction parameters were studied to simulate the MV degradation process, including the initial MV concentrations, reaction $\mathrm{pH}$ and catalyst dosages. The study on the photocatalytic mechanism of the $\mathrm{Fe}-\mathrm{C} / \mathrm{N}-900$ composite implies that $\mathrm{O}_{2} \cdot{ }^{-}$should be the major contributor to the degradation process of $\mathrm{MV}$. Last but not least, this study is a good example showing that NPCs can be a competent substrate for photocatalyst used in water treatment.

\section{ACKNOWLEDGEMENTS}

This research was partially funded by the Natural Science Foundation of China (51978415), Science Foundation of Guangdong Medical University (GDMUZ2019008, GDMUM201821), Featured Innovation Project of Guangdong Province (2019KTSCX053) and Special Funds for Scientific Technological Innovation of Undergraduates in Guangdong Province (pdjh2020a0260), Undergraduate Innovation Experiment Project of Guangdong Medical University (ZZDM003, ZYDM011, ZYDM010) and Key scientific research project of Colleges and Universities of Education Department of Guangdong Province (20202ZDZX2046).

\section{REFERENCES}

1. Jiang, D.; Xu, P.; Wang, H.; Zeng, G.; Huang, D.; Chen, M.; Lai, C.; Zhang, C.; Jia, W.; Xue, W. Strategies to improve metal organic frameworks photocatalyst's performance for degradation of organic pollutants. Coord. Chem. Rev. 2018, 376, 449-466.

2. Liu, J.-Q.; Luo, Z.-D.; Pan, Y.; Singh, A.K.; Trivedi, M.; Kumar, A. Recent developments in luminescent coordination polymers: Designing strategies, sensing application and theoretical evidences. Coord. Chem. Rev. 2020, 406, 213-245.

3. Bhadra, N.; Ahmed, I.; Kim, S.; Jhung, S. Adsorptive removal of ibuprofen and diclofenac from water using metal-organic framework-derived porous carbon. Chem. Eng. J. 2017, 314, 50-58.

4. Ahmed, M.; Zhou, J.; Ngo, H.; Guo, W.; Thomaidis, N.; Xu, J. Progress in the biological and chemical treatment technologies for emerging contaminant removal from wastewater: A critical review. J. Hazard. Mater. 2017, 323, 274-298.

5. Hu, M.-L.; Razavi, S.A.; Piroozzadeh, M.; Morsali, A. Sensing organic analytes by metalorganic frameworks: A new way of considering the topic. Inorg. Chem. Front. 2020, 7, 1598-1632.

6. Esrafili, L.; Firuzabadi, F.D.; Morsali, A.; Hu, M.L. Reuse of predesigned dual-functional metal organic frameworks (DF-MOFs) after heavy metal removal. J. Hazard. Mater. 2021, 403, 123696.

7. Kubacka, A.; Fernándezgarcía, M.; Colón, G. Advanced nanoarchitectures for solar photocatalytic applications. Chem. Rev. 2012, 112, 1555-1614.

8. Su, C.; Goforth, A.; Smith, M.; Pellechia, J.; Zur-Loye, H. Exceptionally stable, hollow tubular metal-organic architectures: synthesis, characterization, and solid-state transformation study. J. Am. Chem. Soc. 2004, 126, 3576-3586.

9. Liu, W.; Shen, X.; Han, Y.; Liu, Z.; Dai, W.; Dutta, A.; Kumar, A.; Liu, J. Selective adsorption and removal of drug contaminants by using an extremely stable $\mathrm{Cu}(\mathrm{II})$-based 3D metal-organic framework.Chemosphere 2019, 215, 524-531. 
10. Jiang, H.; Liu, B.; Lan, Y.; Kuratani, K.; Akita, T.; Shioyama, H.;Zong, F.; Xu, Q. From metal-organic framework to nanoporous carbon: toward a very high surface area and hydrogen uptake. J. Am. Chem. Soc. 2011, 133, 11854-11857.

11. Bhadra, B.; Ahmed, I.; Kim, S.; Jhung, S. Adsorptive removal of ibuprofen and diclofenac from water using metal organic framework-derived porous carbon. Chem. Eng. J. 2017, 314, 50-58.

12. Feng, D.; Gu, Z.; Li, J.; Jiang, H.; Wei, Z.; Zhou, H. Zirconium-metalloporphyrin PCN-222: Mesoporous metal-organic frameworks with ultrahigh stability as biomimetic catalysts. Angew. Chem. 2012, 51, 10307-10310.

13. Yuan, X.; Jiang, L.; Chen, X.; Leng, L.; Wang, H.; Wu, Z.; Xiong, T.; Liang, J.; Zeng, G. Highly efficient visible-light-induced photoactivity of Z-scheme $\mathrm{Ag}_{2} \mathrm{CO}_{3} / \mathrm{Ag} / \mathrm{WO}_{3}$ photocatalysts for organic pollutant degradation. Environ. Sci.: Nano. 2017, 4, 2175-2185.

14. Pan, Y.; Yuan, X.; Jiang, L.; Wang, H.; Yu, H.; Zhang, J. Stable self-assembly AgI/UiO$66\left(\mathrm{NH}_{2}\right)$ heterojunction as efficient visible-light responsive photocatalyst for tetracycline degradation and mechanism insight. Chem. Eng. J. 2020, 384, 123310-123326.

15. Wu, Z.B.; Yuan, X.Z.; Zhang, J.; Wang, H.; Jiang, L.B.; Zeng, G.M. Photocatalytic decontamination of wastewater containing organic dyes by metal-organic frameworks and their derivatives. Chem CatChem. 2017, 9, 41-64.

16. Ye, S.; Yan, M.; Tan, X.; Liang, J.; Zeng, G.; Wu, H.; Song, B.; Zhou, C.; Yang, Y.; Han, W. Facile assembled biochar-based nanocomposite with improved graphitization for efficient photocatalytic activity driven by visible light. Appl. Catal. B Environ. 2019, 250, 78-88.

17. Zhang, M.; Wang, L.; Zeng, T.; Shang, Q.; Zhou, H.; Pan, Z.; Cheng, Q. Two pure MOFphotocatalysts readily prepared for the degradation of methylene blue dye under visible light. Dalton Trans. 2018, 47, 4251-4258.

18. Jiang, L.; Yuan, X.; Zeng, G.; Liang, J.; Wu, Z.; Wang, H.; Zhang, J.; Xiong, T.; Li, H. A facile band alignment of polymeric carbon nitride isotype heterojunctions for enhanced photocatalytic tetracycline degradation. Environ. Sci.: Nano. 2018, 5, 2604-2617.

19. Yuan, F.; Yuan, C.; Zhou, C.; Qiao, C.; Lu, L.; Ma, A.; Singh, A.; Kumar, A. Syntheses and photocatalytic properties of three new $\mathrm{d}^{10}$-based coordination polymers: effects of metal centers and ancillary ligands. CrystEngComm. 2019, 21, 6558-6565.

20. Liu, L.; Yu, C.; Du, J.; Liu, S.; Cao, J.; Ma, L. Construction of five $\mathrm{Zn}(\mathrm{II}) / \mathrm{Cd}(\mathrm{II})$ coordination polymers derived from a new linear carboxylate/pyridyl ligand: Design, synthesis, and photocatalytic properties. Dalton Trans. 2016, 45, 12352-12361.

21. Pan, Y.; Ding, Q.; Xu, H.; Shi, C.; Singh, A.; Kumar, A.; Liu, J. A new Zn(II)-based 3D metal-organic framework with uncommon sev topology and its photocatalytic properties for the degradation of organic dyes. CrystEngComm. 2019, 21, 4578-4585.

22. Wang, C.; Li, J.; Lv, X.; Zhang, Y.; Guo, G. Photocatalytic organic pollutants degradation in metal-organic frameworks. Energy Environ. Sci. 2014, 7, 2831-2867.

23. Ding, Q.; Pan, Y.; Luo, Y.; Zhou, M.; Guan, Y.; Li, B.; Trivedi, M.; Kumar, A.; Liu, J. Photocatalytic and ferric ion sensing properties of a new three-dimensional metal-organic framework based on cuboctahedral secondary building units. ACS Omega 2019, 4, 1077510783.

24. Wang, F.; Wang, C.; Yu, Z.; Xu, K.; Li, X.; Fu, Y. Two multifunctional Mn(II) metalorganic frameworks: Synthesis, structures and applications as photocatalysis and luminescent sensor. Polyhedron 2016, 105, 49-55.

25. Hu, M.-L.; Safarifard, V.; Doustkhah, E.; Rostamnia, S.; Morsali, A.; Nouruzi, N.; Beheshti, S.; Akhbari, K. Taking organic reactions over metal-organic frameworks as heterogeneous catalysis. Micropor. Mespor. Mater. 2018, 256, 111-127. 